\title{
Dust and molecules in extra-galactic planetary nebulae
}

\author{
D. A. García-Hernández ${ }^{1,2} \dagger$ \\ ${ }^{1}$ Instituto de Astrofísica de Canarias, C/ Via Láctea s/n, E-38205 La Laguna, Spain \\ email: agarcia@iac.es \\ ${ }^{2}$ Departamento de Astrofísica, Universidad de La Laguna (ULL), E-38206 La Laguna, Spain
}

\begin{abstract}
Extra-galactic planetary nebulae (PNe) permit the study of dust and molecules in metallicity environments other than the Galaxy. Their known distances lower the number of free parameters in the observations vs. models comparison, providing strong constraints on the gasphase and solid-state astrochemistry models. Observations of PNe in the Galaxy and other Local Group galaxies such as the Magellanic Clouds (MC) provide evidence that metallicity affects the production of dust as well as the formation of complex organic molecules and inorganic solid-state compounds in their circumstellar envelopes. In particular, the lower metallicity $\mathrm{MC}$ environments seem to be less favorable to dust production and the frequency of carbonaceous dust features and complex fullerene molecules is generally higher with decreasing metallicity. Here, I present an observational review of the dust and molecular content in extra-galactic $\mathrm{PNe}$ as compared to their higher metallicity Galactic counterparts. A special attention is given to the level of dust processing and the formation of complex organic molecules (e.g., polycyclic aromatic hydrocarbons, fullerenes, and graphene precursors) depending on metallicity.
\end{abstract}

Keywords. Astrochemistry, molecular processes, Planetary nebulae, AGB and post-AGB, dust.

\section{Introduction}

At the end of the asymptotic giant branch (AGB) phase, low- and intermediate-mass stars experience thermal pulses and strong mass loss. The strong mass loss efficiently enriches the interstellar medium (ISM) with specific isotopes and dust grains. The main processes of nucleosynthesis take place during the thermal pulsing phase on the AGB, while the molecular processes are more important in the transition phase between AGB stars and planetary nebulae (PNe).

During the thermal pulsing phase on the AGB, ${ }^{12} \mathrm{C}$ and heavy s-process elements such as $\mathrm{Rb}, \mathrm{Zr}$, etc. are produced and dredge-up to the stellar surface. At solar metallicity, low-mass AGB stars $\left(\mathrm{M}<1.5 \mathrm{M}_{\odot}\right)$ are O-rich and they probably do not form PNe. Intermediate-mass AGBs $\left(1.5<\mathrm{M}<4 \mathrm{M}_{\odot}\right)$ are $\mathrm{C}$-rich and they do not experience hot bottom burning $(\mathrm{HBB})$, while the high-mass $\left(\mathrm{M}>4 \mathrm{M}_{\odot}\right)$ AGB stars remain O-rich because of the HBB activation. This evolutionary scenario has a strong dependence with metallicity (e.g., García-Hernández et al. 2006, 2007, 2009 and references therein). For example, the mass limit for HBB activation decreases with decreasing metallicity. In short, the more massive AGB stars produce different elements than lower mass AGBs and this should be reflected in the gas and circumstellar dust chemistry.

AGB stars are also wonderful molecular factories and more than 60 molecules have been detected in the circumstellar envelopes around AGB stars (e.g., Herbst \& van Dishoeck 2009). These molecules are mainly detected via their rotational transitions from the

$\dagger$ D.A.G.H. acknowledges support provided by the Spanish Ministry of Economy and Competitiveness (MINECO) under grants AYA-2011-27754 and AYA-2014-58082-P. 

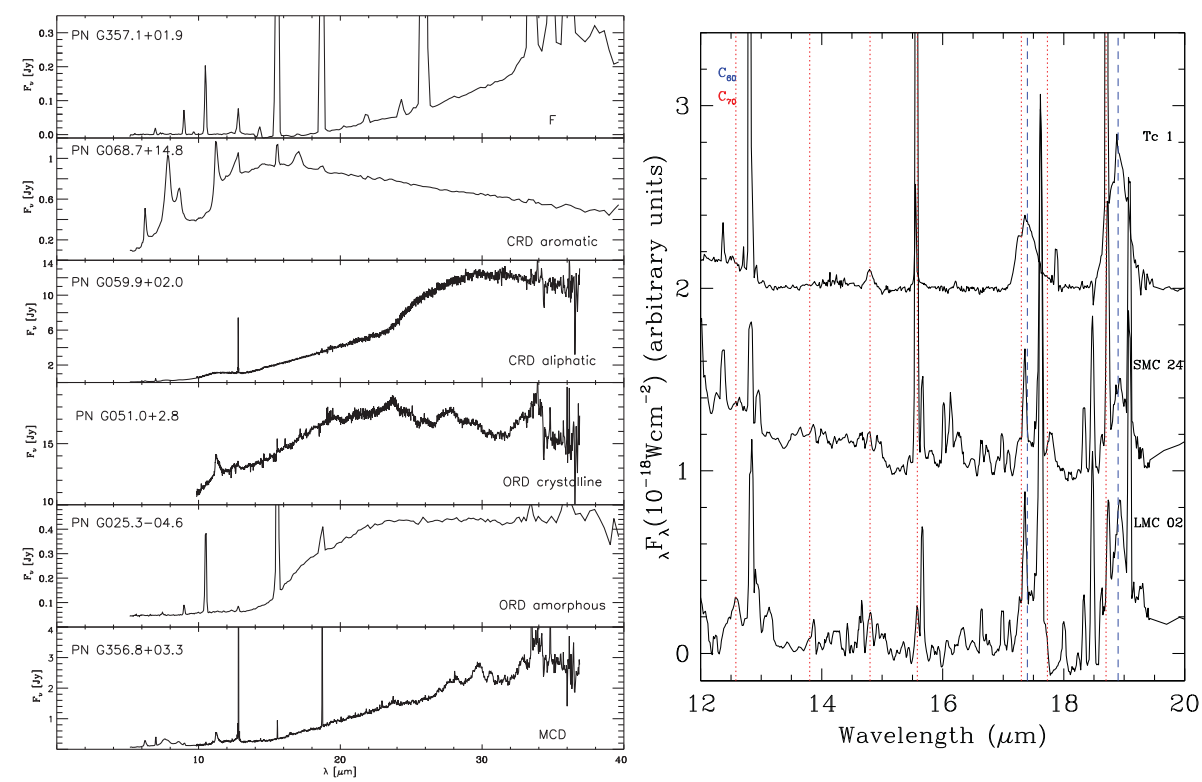

Figure 1. Left panel: Spitzer spectral templates of the several dust types (from top to bottom: featureless $(\mathrm{F})$; carbon-chemistry aromatic or aliphatic $\left(\mathrm{CC}_{a r}\right.$ or $\left.\mathrm{CC}_{a l}\right)$, oxygen-chemistry crystalline or amorphous $\left(\mathrm{OC}_{c r}\right.$ or $\left.\mathrm{OC}_{a m}\right)$, and double-chemistry (DC)), found in compact Galactic PNe (updated from Stanghellini et al. 2012). Right panel: Residual Spitzer spectra ( 10-23 $\mu \mathrm{m}$ ) for the $\mathrm{C}_{70} \mathrm{MC}$ PNe LMC 02 and SMC 24 in comparison with the Galactic PN Tc 1. The $\mathrm{C}_{60}$ (dashed) and $\mathrm{C}_{70}$ (dotted) band positions are marked (udapted from García-Hernández et al. 2011).

infrared (IR) to milimeter wavelengths. They are mainly gas-phase molecules, including: inorganics such as $\mathrm{SiO}, \mathrm{SiS}$, and $\mathrm{NH}_{3}$, organics like $\mathrm{C}_{2} \mathrm{H}_{2}$ and $\mathrm{CH}_{4}$, radicals (e.g., $\mathrm{HCO}^{+}$), rings (e.g., $\mathrm{C}_{3} \mathrm{H}_{2}$ ) or chains (e.g., $\mathrm{HC}_{9} \mathrm{~N}$ ). Note that gas-phase reactions can explain most of these molecules but not all of them and solid-state chemistry has to be considered in the models; e.g., molecules may form on dust grains.

The composition of the dust around evolved stars depends on the dominant stellar chemistry. The O-rich AGB stars display amorphous silicates, weak crystalline silicates like olivine and piroxenes, and refractory oxides such as corundum and spinel (e.g., Waters et al. 1996), while the C-rich AGBs show silicon carbide and amorphous carbon, but other complex organic compounds with mixed aromatic/aliphatic structures (e.g., kerogen) may provide also the strong dust continuum emission (e.g., Kwok 2004). On the other hand, young and evolved PNe are mainly characterized by the aromatic infrared bands (usually associated with polycyclic aromatic hydrocarbons; PAHs) and unidentified infrared emission (UIR) and crystalline silicates for the case of a C-rich and O-rich chemistry, respectively. PNe showing both C-rich and O-rich dust features, what we call mixed-chemistry, are also observed.

PNe in our own Galaxy are characterized by a strong IR dust continuum emission and the following Spitzer Space Telescope dust types can be defined depending on the nature of the solid-state features atop of the continuum (Stanghellini et al. 2012; García-Hernández \& Górny 2014): i) Featureless (F) PNe with only nebular emission lines in their spectra and little dust continuum; ii) Carbon chemistry (CC) PNe with C-rich dust features that can be aromatic and/or aliphatic; iii) Oxygen chemistry (OC) PNe with O-rich dust features that can be crystalline and/or amorphous; iv) Double-chemistry (DC) PNe with mixed C-rich and O-rich dust features that can be crystalline and/or amorphous. Figure 1 
Table 1. Dust type statistics in different metallicity environments.

\begin{tabular}{|l|c|c|c|}
\hline Dust type & $\begin{array}{c}\text { Galactic Bulge } \\
\text { high z (50) }^{1}\end{array}$ & $\begin{array}{c}\text { Galactic Disk } \\
\text { intermediate z (98) }\end{array}$ & $\begin{array}{c}\text { Magellanic Clouds } \\
\text { low z (66) }\end{array}$ \\
\hline F & $14 \%(7)$ & $19 \%(18)$ & $41 \%(27)$ \\
CC & $8 \%(4)$ & $33 \%(32)$ & $52 \%(34)$ \\
OC & $26 \%(13)$ & $31 \%(31)$ & $7 \&(5)$ \\
DC & $52 \%(26)$ & $18 \%(17)$ & $0 \%(0)^{2}$ \\
\hline Ref. $^{3}$ & S12, GH14 & S12, GH14 & S07, BS09 \\
\hline
\end{tabular}

Notes:

${ }^{1}$ Total number of PNe in each metallicity environment.

${ }^{2}$ The LMC and SMC PNe show very similar distributions among the dust types.

${ }^{3}$ References for the dust types. S12: Stanghellini et al. (2012); GH14: García-Hernández \& Górny (2014); S07:

Stanghellini et al. (2007); BS09: Bernard-Salas et al. (2009).

(left panel) displays several Spitzer spectra of compact Galactic PNe with different dust types. There is an aromatization or crystallization process from AGB stars to PNe and the aromatic (C-rich) and crystalline (O-rich) compounds are synthesized during this short transition phase (García-Hernández 2012 and references therein). Thus, post-AGB stars or proto-PNe are wonderful laboratories for astrochemistry because they provide strong constraints on the gas-phase and solid-state chemical models. A major challenge is to understand the formation pathways of these complex organic molecules and inorganic solid-state compounds.

PNe have been detected in sixteen Local Group members. There are dwarf galaxies nearer to the Milky Way but only a few PNe have been found in them (e.g., Reid 2012). The Magellanic Clouds (MCs) provide single environments at known distances with higher luminosities than those of the closer galaxies, being an excellent laboratory for the AGB theories and the molecular and dust formation models. In addition, the MCs offer lower metallicity environments than our own Galaxy. Its relative vicinity provide us with the most complete PNe sample (and data) with the extra of a low-extinction. In particular, the well known distances lower the number of free parameters in the comparison between observations and the models. The AGB nucleosynthesis (and so the dust formation) strongly depend on metallicity, something that should be reflected in the gas and dust composition and that we can test by studying PNe in the MCs.

\section{PNe dust types versus metallicity}

The several Spitzer dust types in PNe vary with the metallicity of the environment. This is shown in Table 1, where the dust type statistics for low-metallicitiy MC PNe are presented in comparison with other Galactic environments such as the Galactic Disk (intermediate metallicity) and Bulge (high metallicity). The dust features atop the strong dust continua are more frequent in metal-rich environments like the Galactic Disk and Bulge, as expected. Within the PNe with solid-state features, carbon chemistry (CC) is predominant in the MCs, where the crystalline silicates and other O-rich dust features are very rare (Stanghellini et al. 2007). Also, double-chemistry (DC) is not observed in the MCs. The O-rich (OC) and C-rich (CC) objects are equally predominant in Galactic Disk PNe (Stanghellini et al. 2012; García-Hernández \& Górny 2014), but the Galactic Bulge PNe show a majority of double-chemistry (DC) dust types (Perea-Calderón et al. 2009).

Oxygen-rich chemistry PNe. The O-rich chemistry PNe are less common at the low metallicity of the MCs (Stanghellini et al. 2007) and the statistics of the Spitzer dust subtypes (amorphous or crystalline) change with the metallicity of the environment (e.g., 
García-Hernández \& Górny 2014). For example, the amorphous silicate features in emission at $\sim 9.7 \mu \mathrm{m}$ are more frequent at the intermediate metallicities of the Galactic Disk, while the crystalline silicate features (e.g., at 23.5, 27.5 and $33.5 \mu \mathrm{m}$ ) are very rare in the MCs but they completely dominate at the high metallicity of the Galactic Bulge.

Carbon-rich chemistry PNe. In the case of the C-rich PNe, just the opposite is seen and they are more frequent at the MC low-metallicity (Table 1). The C-rich MC PNe usually show less processed dust grains or small dust grains (Stanghellini et al. 2007); the aliphatic dust dominates the MC PNe Spitzer spectra and the PAHs are not very frequent. Also, the frequency of C-rich PNe varies with the environment, dominating in the MCs and being absent in the Galactic Bulge (Table 1). The PNe with aliphatic dust features such as those at $\sim 9-13,15-20$, and $25-35 \mu \mathrm{m}$ (see Section 3 ) are very frequent in the MCs, while their Galactic Disk counterparts are usually compact (and presumably young) PNe of sub-solar metallicity (García-Hernández \& Górny 2014). At solar metallicity, the strong dust continuum emission and the $\sim 11.5 \mu \mathrm{m} \mathrm{SiC}$ feature seen in the AGB phase evolve to small hydrocarbon molecules (such as $\mathrm{C}_{2} \mathrm{H}_{2}$ ) in absorption together with broad $30 \mu \mathrm{m}$ emission in the post-AGB stage. The dust features evolve very quickly from aliphatics to PAHs and when the central star is hot enough to form a PN, the IR spectrum is dominated by nebular emission lines and we still can see the $30 \mu \mathrm{m}$ feature (García-Lario \& Perea-Calderón 2003). The IR spectral evolution is a little bit different at the lower metallicity of the MCs and the transition from aliphatic dust features such as the $\sim 9-13 \mu \mathrm{m}$ feature to PAHs seems to be slower (Stanghellini et al. 2007). There exist two main aromatization models from the AGB phase to the PN stage: i) the dust processing by the UV photons from the central star, which changes the structure from aliphatic to aromatic (e.g., Kwok et al. 2001; García-Lario \& PereaCalderón 2003); or ii) $\mathrm{C}_{2} \mathrm{H}_{2}$ and its radical derivatives are the precursors of PAHs (e.g., Cernicharo et al. 2004).

\section{The unidentified 21,26 , and $30 \mu \mathrm{m}$ features}

There is an interesting group of still UIR features located at $\sim 21,26$, and $30 \mu \mathrm{m}$, which are only observed in C-rich stars evolving from the AGB phase to the PN stage.

The $21 \mu \mathrm{m}$ feature is usually observed in proto-PNe, indicating that its carrier is of solid-state origin with a fragile character (see also Section 4). On the other hand, the $30 \mu \mathrm{m}$ feature (sometimes with substructure at $\sim 26 \mu \mathrm{m}$ ) is seen all the way from AGB stars to PNe, suggesting that its carrier is very abundant in the circumstellar envelope. Many carriers for this specific UIR features have been proposed to date. For example, nanodiamonds, hydrogenated fullerenes (fullerAnes), and hydrogenated amorphous carbon grains (HACs) have been linked to the $21 \mu \mathrm{m}$ feature, while the $30 \mu \mathrm{m}$ feature could be explained by magnesium sulfide $(\mathrm{MgS})$, aliphatic chains, or HACs (García-Hernández 2012 and references therein). It is very interesting that the identification of these UIR features as HAC like-dust (some kind of solid with a mixed aromatic/aliphatic structure) may explain the detection of complex molecules such as fullerenes and planar $\mathrm{C}_{24}$ (graphene precursors) in some C-rich PNe (see below).

\section{Complex organic molecules versus metallicity}

Fullerenes such as $\mathrm{C}_{60}$ and $\mathrm{C}_{70}$ are very stable molecules. These complex organic molecules are very important for interstellar/circumstellar chemistry because they may explain many astrophysical phenomena like the diffuse interstellar bands (DIBs) and the ultraviolet bump. Fullerenes were discovered in the laboratory by Kroto et al. (1985) 
and they are also found on Earth and meteorites. $\mathrm{C}_{60}{ }^{+}$was also tentatively detected in the ISM (Foing \& Ehrenfreund 1994), which has been recently confirmed by laboratory spectroscopy (Campbell et al. 2015). At laboratory, fullerenes are efficiently produced under H-poor conditions and in 2010 Cami et al. reported the first IR detection of neutral $\mathrm{C}_{60}$ and $\mathrm{C}_{70}$ in the young PN Tc 1 (which shows a lack of PAH features in its Spitzer spectrum) as due to the H-poor conditions in the circumstellar envelope.

Fullerenes in Galactic H-rich PNe. We now know that fullerenes can be detected together with PAHs and that they are efficiently formed in H-rich circumstellar envelopes only (García-Hernández et al. 2010; García-Hernández, Rao \& Lambert 2011). Fullerenes are detected in Galactic PNe with normal $\mathrm{H}$ abundances and this result is confirmed by the independent detection of $\mathrm{C}_{60}$ in only those $\mathrm{R}$ Coronae Borealis stars with some $\mathrm{H}$. Remarkably, fullerene PNe display broad HAC-like dust features at $\sim 9-13$ and $25-35$ $\mu \mathrm{m}$, suggesting that both fullerenes and PAHs probably evolve from the photochemical processing of HACs, as indicated by some laboratory experiments (Scott et al. 1997). Indeed, fullerenes have been detected also in other Galactic objetcs such as a proto-PN, reflection nebulae, and young stellar objects, and none of these space environments is H-poor (see e.g., García-Hernández 2012 and references therein).

Extra-galactic fullerenes. Fullerenes have been detected in PNe of the MCs and the first extra-galactic detection of $\mathrm{C}_{70}$ was reported (García-Hernández et al. 2011). Figure 1 (right panel) shows the $\mathrm{C}_{60}$ and $\mathrm{C}_{70}$ emission features detected in MC PNe. The combination of the Spitzer spectra with laboratory data permitted the determination of accurate abundances of both $\mathrm{C}_{60}(0.07 \%)$ and $\mathrm{C}_{70}(0.03 \%)$. The great variety of molecular species (HACs, PAHs clusters, fullerenes, etc.) observed in MC PNe seems to support the HACs scenario proposed by García-Hernández et al. (2010), where fullerenes may evolve from the UV-induced HACs decomposition (see also Micelotta et al. 2012).

Fullerenes detection vs. metallicity. Interestingly, the detection rate of fullerenes in Crich PNe increases with decreasing metallicity; $\sim 5 \%$ of fullerene $\mathrm{PNe}$ are found in our Milky Way, while the fullerenes detection rate is $\sim 20 \%$ and $\sim 44 \%$ in the LMC and SMC, respectively (García-Hernández et al. 2012). This suggests a more limited dust processing (or the general presence of small dust grains) at low metallicity. Indeed, Otsuka et al. (2014) have shown that all Galactic fullerene PNe are sub-solar metallicity low-mass $\mathrm{PNe}$, which demonstrate that low metallcity environments favours fullerene production and detection.

In addition, the $21 \mu \mathrm{m}$ feature is more common in the MCs than in the Galaxy (Volk et al. 2011) and its carrier may be related to the formation of fullerenes. Volk et al. (2011) reported an anti-correlation between the $30 \mu \mathrm{m}$ and other UIR features for the MC 21 $\mu \mathrm{m}$ sources. Such an anti-correlation could result from radiation-induced decomposition of HAC grains into PAHs and fullerenes. Note that in the HACs scenario the $21 \mu \mathrm{m}$ feature is also related with the formation of fullerenes and its carrier may be a fragile intermediate product from the decomposition of HAC or a similar material. However, a more recent study on a smaller sample of Galactic proto-PNe claims that for the Galactic $21 \mu \mathrm{m}$ sources the $30 \mu \mathrm{m}$ and the UIR features do not seem to be anti-correlated (Mishra et al. 2015). It is to be noted here that spectroscopically the MC $21 \mu \mathrm{m}$ sources differ from the Galactic ones, with the MC $21 \mu \mathrm{m}$ sources displaying more typical UIR features (i.e., more PAH-like).

Graphene precursors in PNe. Unusual emission features at $\sim 6.6,9.8$, and $20 \mu \mathrm{m}$ have been also detected in MC PNe (García-Hernández et al. 2011). These features are coincident with the theoretical transitions of the planar $\mathrm{C}_{24}$ molecule (just a piece of graphene or a graphene precursor). However, a confirmation has to wait for laboratory 
spectroscopy, which is extremely difficult because of the high reactivity of $\mathrm{C}_{24}$. The most interesting point here is that the possible detection of graphene precursors $\left(\mathrm{C}_{24}\right)$ opens the possibility of detecting other forms of $\mathrm{C}$ in space. For example, fullerenes and PAHs may coexist in fullerene $\mathrm{PNe}$ and $\mathrm{C}_{60}$ can react with a small $\mathrm{PAH}$ like anthracene via Diels-Alder reactions to form fullerene/anthracene mono-adducts or bis-adducts. Such fullerene/PAHs adducts display the same mid-IR features of isolated $\mathrm{C}_{60}$ molecules and we could not distinguish them via mid-IR spectroscopy only (García-Hernández, Cataldo \& Manchado 2013).

\section{Concluding remarks}

In summary, the different dust properties (and evolution) at different metallicities (MCs, Galactic Disk and Bulge) are consistent with the AGB nucleosynthesis theoretical predictions (third dredge-up and HBB). The coexistence of PAHs, fullerenes, HACs, and possible planar $\mathrm{C}_{24}$ (graphene precursosr) suggests a top-down scenario for the fullerenes formation. The dust processing is more limited at the low metallicity of the MCs, which otherwise provide the most favorable conditions for fullerene formation and detection. A complex family of fullerene-based molecules (e.g., fullerene/PAH adducts, metallofullerenes, etc.) is likely to be present in space but more laboratory efforts are needed.

\section{References}

Bernard-Salas, J. et al. 2009, ApJ, 699, 1541

Cami, J. et al. 2010, Science, 329, 1180

Campbell, E. K., Holz, M., Gerlich, D., \& Maier, J. P. 2015, Nature, 523, 322

Cernicharo, J. 2004, ApJ, 608, L41

Foing, B. H. \& Ehrenfreund, P. 1994, Nature, 369, 296

García-Lario, P. \& Perea-Calderón, J. V. 2003, ESA Publication Series, 511, 97

García-Hernández, D. A. et al. 2006, Science, 314, 1751

García-Hernández, D. A. et al. 2007, A\&A, 462, 711

García-Hernández, D. A. et al. 2009, ApJ, 705, L31

García-Hernández, D. A. et al. 2010, ApJ, 724, L39

García-Hernández, D. A. et al. 2011, ApJ, 737, L30

García-Hernández, D. A., Rao, N. K., \& Lambert, D. L. 2011, ApJ, 729, 126

García-Hernández, D. A. et al. 2012, ApJ, 760, 107

García-Hernández, D. A. 2012, in IAU Symp. 283, Cambridge Univ. Press, 148

García-Hernández, D. A., Cataldo, F., \& Manchado, A. 2013, MNRAS, 434, 415

García-Hernández, D. A. \& Górny, S. K. 2014, A\&̋A, 567, A12

Herbst, E. \& van Dishoeck, E. F. 2009, ARA\& $\&$, 47, 427

Kroto, H. W. et al. 1985, Nature, 318, 162

Kwok, S. et al. 2001, ApJ, 554, L87

Kwok, S. 2004, Nature, 430, 985

Micelotta, E. R. et al. 2012, ApJ, 761, 35

Mishra, A., Li, A., \& Jiang, B. W. 2015, ApJ, 802, 39

Otsuka, M. et al. 2014, MNRAS, 437, 2577

Perea-Calderón, J. V. et al. 2009, A\& A, 495, L5

Reid, W. A. 2012, in IAU Symp. 283, Cambridge Univ. Press, 227

Scott, A., et al. 1997, ApJ, 489, L123

Stanghellini, L. et al. 2007, ApJ, 671, 1669

Stanghellini, L. et al. 2012, ApJ, 753, 172

Volk, K. et al. 2011, ApJ, 735, 127

Waters, L. B. F.. M. et al. 1996, A\& A, 315, L361 